\title{
Systematic Analysis of Parameter Identifiability for Improved Fitting of a Biological Wastewater Model to Experimental DatađI
}

\author{
CRISTINA SARMIENTO FERRERO $\dagger$, QIAN CHAI $\dagger$, \\ MARTA DUEÑAS DÍEZ $\ddagger$ SVERRE H. AMRANI§ and BERNT LIE*†
}

Keywords: ASM2d, identifiability analysis, parameter estimation, model fitting

In this paper, a general description of wastewater treatment based on activated sludge is given, with emphasis on the ASM2d model. Particular emphasis has been given to make the presentation readable without a too detailed prior knowledge of wastewater treatment. Next, a method for experimental parameter identifiability analysis is described. After a presentation of the wastewater treatment plant at Duvbacken in Gävle, Sweden, the experimental identifiability of the dynamic model is analyzed. Out of the 45 original parameters in the model, the analysis indicates that with the given experimental conditions, 12 parameters can be identified.

\section{Introduction}

Water is one of our most precious resources, and wastewater treatment is becoming more and more important in a highly populated, industrialized world. Wastewater contains organic matter and microorganisms, and the microorganisms thus deplete the water for oxygen. Furthermore, nitrogen and phosphorous content leads to algae growth. It is thus of importance to reduce the content of organic matter, as well as nitrogen and phosphorous. There are also safety regulations on the content of microorganisms, as well as on other content in the wastewater. See e.g. Metcalf \& Eddy (2003) for an overview of characteristics of wastewater, and treatment of wastewater.

Both for design and operation of wastewater treatment plants, it is of interest to develop models of how the plants transform the feed. One characteristic of wastewater feed is its highly varying flow rate and composition (Olsson \& Newell 1999). This implies that dynamic models are highly relevant for wastewater treatment plants. One such series of models are the Activated Sludge Models (ASM) (Henze, Harremoës, la Cour Jansen \& Arvin 1996), which have been developed to include a description of organic content and decomposition, microorganisms, nitrogen processes, and phosphorous processes. In particular, ASM2d attempts to describe the important processes involved in the transformation of organic matter, nitrogen, and phosphorous (Henze, Gujer, van Loosdrecht \& Mino 2000).

The ASM models are parametric, and nominal parameter values are suggested in publications. However, the models are based on averages of microorganism and organic matter populations, and wastewater characteristics vary from plant to plant. It is thus necessary to fit the model parameters to experimental data from a specific wastewater

*Corresponding author: Bernt.Lie@ @it.no.

$\dagger$ Telemark University College, P.O. Box 203, N-3901 Porsgrunn, Norway.

$\$$ Hydro Corporate Research Center, Porsgrunn, Norway.

$\S$ BioTek AS, Porsgrunn, Norway.

IIAn early version of this paper was presented at SIMS 2005, the 46th Conference on Simulation and Modeling, Trondheim, Norway, October 13-14, 2005. 
stream in order to ensure good predictive properties of a model (Jeppson 1993), (Brun, Kühni, Siegrist, Gujer \& Reichert 2002).

In this paper, we consider the Duvbacken wastewater treatment plant in Gävle, Sweden, which is designed to treat the municipal wastewater from $100.000 \mathrm{pe}^{1}$. This plant has recently been modified to remove phosphorous, nitrogen, and organic matter, primarily using bacteria. In situations where the biology represented by the bacteria struggle to perform according to governmental effluent criteria, chemicals are added as a supplement. The particular emphasis is to study the identifiability of parameters in a dynamic model of the plant, based on available experimental data. In this paper, real experimental data were unavailable, and the study is therefore based on simulated data. However, the procedure can be used without modification with real data.

In section 2 of the paper, an overview of principles for modeling biological wastewater treatment (BWWT) is given. In section 3, basic principles of parameter identifiability are discussed. In section 4, a plant at Duvbacken, Gävle, is presented, and a model of the plant is verified. In section 5, identifiability analysis for the Duvbacken model is carried out, and some results of model fitting are given. Finally, some conclusions are drawn.

\section{Biological Wastewater Treatment}

\subsection{Wastewater characterization and treatment}

Due to the importance of clean water, wastewater treatment is a large and growing industry throughout the world. Microorganisms in water tend to use dissolved oxygen (DO) while consuming organic and inorganic compounds, resulting in harmfully low concentrations of DO to the aquatic environment. Thus, a primary task in wastewater treatment is to remove the oxygen consuming pollutants and their feed. Other pollutants consisting of nitrogen and phosphorous lead to eutrophication, which is the accelerated ageing of lakes and estuaries due to excessive plant and algal growth. It is thus in addition important to remove nitrogen and phosphorous compounds from wastewater.

The composition of wastewater depends on its source; important classes of wastewaters are industrial and domestic wastes. The latter, sewage, consists of substances such as ground garbage, laundry water, and excrement. More than $99 \%$ of wastewater is water, and about $0.5 \mathrm{~g} / \mathrm{l}$ is suspended solids such as cellulose and organic matter in the form of fatty acids, carbohydrates, and proteins. The bad odor of sewage stems from protein decomposition under anaerobic ${ }^{2}$ conditions. Sewage contains a varied population of microorganisms such as bacteria, which must not be mixed into drinking water. Still, microorganisms are useful in transforming dissolved substances into particulate substances which can then be removed from the wastewater.

As mentioned, one problem with wastewater is that it contains carbonaceous matter which through chemical reactions depletes water for oxygen. The simplest measure of the content of such matter, is the biochemical oxygen demand (BOD): the BOD is equal to the amount of dissolved oxygen that is consumed by the wastewater when incubated for a specified length of time at $20^{\circ} \mathrm{C}$, typically for 5 or 7 days $\left(\mathrm{BOD}_{5}\right.$ or $\mathrm{BOD}_{7}$, respectively); $\mathrm{BOD}$ measures the oxidation of organic matter. A better measure is the

\footnotetext{
${ }^{1} \mathrm{pe}=$ person equivalents.

${ }^{2}$ Anaerobic, i.e., without the presence of oxygen or oxides.
} 
Table 1. Typical composition of domestic waste water, with indication of effluent standards. From Bailey \& Ollis (1986).

Parameter

Influent raw wastewater

Effluent in an acceptable plant

BOD, mg/l

COD, mg/l

Suspended solids, $\mathrm{mg} / \mathrm{l}$

Nitrogen, mg/l

Total phosphorous, $\mathrm{mg} / \mathrm{l}$

$100-250$
$200-700$
$100-400$
$20-30$
$6-10$

$5-15$

$15-75$

$10-25$

$2-5$

$0.2-0.6$

chemical oxygen demand (COD), both because it can be measured routinely in a couple of hours ${ }^{3}$ (Bailey \& Ollis 1986), but also because it gives a more accurate measure of the carbonaceous content: COD measures the oxidation of both organic and inorganic matter.

Other undesirable components in wastewater are nitrogen, typically in the form of ammonia $\left(\mathrm{NH}_{3}\right)$, and phosphorous. In addition, wastewater holds both larger objects and readily settleable solids, as well as oil films and other components such as heavy metals and toxic organics. Table 1 illustrates typical feed compositions and effluent standards for sewage.

A wastewater treatment plant typically consists of two or three stages. In the primary stage, easily separable particles (boulders-floc particles, etc.) are removed. In the secondary stage, suspended particles and soluble materials are removed, while in the tertiary stage, remaining contaminants (subcolloidal and soluble materials) are removed, the water is treated chemically to remove bacteria, etc.

In this paper, we will concentrate on the secondary stage, where the wastewater has a muddy consistence and where oxygen is added. Under such oxygenated conditions, microorganisms thrive, forming an active suspension of biological solids (mostly bacteria) called an activated sludge.

\subsection{Wastewater species}

Essentially, microorganisms metabolize and breed while consuming substrate ${ }^{4}$. In this process, the microorganisms synthesize and secrete a polysaccharide gel causing the microbes to agglomerate into flocs (Bailey \& Ollis 1986). These flocs have a high affinity for suspended matter (substrate, etc.), and flocculation thus enables the removal of microorganisms and substrate through sedimentation.

Wastewater contains a large number of microorganisms and substrate, and it is necessary to simplify the description of the components. In the sequel, the components that are detailed are those used in the Activated Sludge Model 2d (ASM2d) (Henze, Gujer, Mino, Matsuo, Wentzel, Marais \& van Loosdrecht 1999), (Henze et al. 2000). The structure of the presentation is similar to that of Jeppson (1993), although his is for ASM1. A minor simplification of the presentation here compared to the full ASM2d, is that we neglect the possibility of phosphorous to bind to metal-hydroxides, and the associated chemical processes. In the presentation, we will follow the naming conventions of the Activated Sludge Models (ASM), which distinguish between dissolved

\footnotetext{
${ }^{3}$ Or in a few minutes using sophisticated instruments.

${ }^{4}$ By substrate is meant nutrients (organic and inorganic matter, including oxygen) and energy.
} 
component $S$ and particulate component $X$. Subscripts are attached to distinguish between the various dissolved and particulate components. As wastewater contains a wide variety of e.g. organic matter, a precise model would need to use population balances. In the ASM, average species are considered in order to simplify the models. In the description, $S$ or $X$ (with subscript) refer both to a specie, and to the concentration of the specie.

Organic substrate is considered to be either biodegradable, or unbiodegradable. The unbiodegradable matter can be either soluble (small species) or particulate (larger species); in both cases, it is considered inert matter, and named $S_{\mathrm{I}}$ and $X_{\mathrm{I}}$, respectively. The biodegradable matter is substrate for the organisms, and can be either soluble (small species/molecules) or particulate (larger species). Particulate biodegradable matter $X_{\mathrm{S}}$ is too large to pass through the cell walls of the microorganisms, and must first be decomposed-which is time consuming, thus $X_{\mathrm{S}}$ is denoted slowly biodegradable substrate. Soluble biodegradable matter is readily biodegradable. ASM2d distinguishes between fermentable biodegradable substrate $\mathrm{S}_{\mathrm{F}}$, and the biodegradable fermentation product $S_{\mathrm{A}}$ (assumed to be acetate $\mathrm{CH}_{3} \mathrm{CO}_{2}^{-}$in later analysis). In ASM1, there was no distinction between $S_{\mathrm{F}}$ and $S_{\mathrm{A}}$, and $S_{\mathrm{S}}=S_{\mathrm{F}}+S_{\mathrm{A}}$ was used there.

Inorganic substrate consists of small molecules, which are soluble. In addition to oxygen $S_{\mathrm{O}_{2}}\left(\mathrm{O}_{2}\right)$, these are nitrous components such as nitrogen $S_{\mathrm{N}_{2}}\left(\mathrm{~N}_{2}\right)$, ammonium $S_{\mathrm{NH}_{4}}$ (some ammonia $\mathrm{NH}_{3}$, but mainly ammonium ion $\mathrm{NH}_{4}^{+}$; assumed to be $100 \%$ ammonium ion in stoichiometric computations), nitrogen oxides $\mathrm{S}_{\mathrm{NO}_{3}}$ (nitrate $\mathrm{NO}_{3}^{-}$and nitrite $\mathrm{NO}_{2}^{-}$; for stoichiometric computations assumed to be $100 \%$ nitrate), and inorganic soluble phosphorous $\mathrm{S}_{\mathrm{PO}_{4}}$ (primarily ortho-phosphates, which are anions of the inorganic chemical compound of phosphoric acid $\mathrm{H}_{3} \mathrm{PO}_{4}$; for stoichiometric computations assumed to consist of $50 \% \mathrm{H}_{2} \mathrm{PO}_{4}^{-}$and $50 \% \mathrm{HPO}_{4}^{2-}$ ).

To facilitate the computation of $\mathrm{pH}$ and charge balance, the alkalinity of wastewater is described by $S_{\mathrm{ALK}}$ (for stoichiometric computations assumed to be bicarbonate $\mathrm{HCO}_{3}^{-}$).

Several types of microorganisms exist in wastewater; all of these are considered to be particulate. Heterotrophic organisms $X_{\mathrm{H}}$ require organic compounds as their substrate; they are responsible for hydrolysis ${ }^{5}$ of slowly biodegradable substrate $X_{\mathrm{S}}$ into smaller components, and grow aerobically ${ }^{6}$ and anoxically ${ }^{7}$, as well as are active in anaerobic $^{8}$ fermentation. Autotrophic organisms $X_{\mathrm{AUT}}$ require inorganic compounds as their substrate; they are responsible for nitrification under aerobic conditions, where ammonium $S_{\mathrm{NH}_{4}}$ is oxidized directly into nitrogen oxides $S_{\mathrm{NO}_{3}}$. Finally, there are phosphate-accumulating organisms (PAO), $X_{\mathrm{PAO}}$; these may grow in both anoxic and aerobic environments.

In addition to the phosphorous accumulating organisms $X_{\mathrm{PAO}}$ mentioned above, two types of cell internal storage products associated with PAO are considered: $X_{\mathrm{PHA}}$ includes primarily organic poly-hydroxyalkanoates (assumed to be poly-hydroxy-butyrate $\left(\mathrm{C}_{4} \mathrm{H}_{6}\right.$ $\left.\mathrm{O}_{2}\right)_{n}$ in stoichiometric computations), and inorganic poly-phosphates $X_{\mathrm{PP}}$ (assumed to be

\footnotetext{
${ }^{5}$ Chemical decomposition of matter by the splitting of a bond and the addition of the hydrogen cation $\mathrm{H}^{+}$and the hydroxide anion $\mathrm{OH}^{-}$of water.

${ }^{6}$ Aerobic $=$ requires the presence of oxygen; $S_{\mathrm{O}_{2}}>0$.

${ }^{7}$ Anoxic $=$ extracts oxygen from nitrate oxides; $S_{\mathrm{O}_{2}} \approx 0, S_{\mathrm{NO}_{3}}>0$. In this process, nitrate $\mathrm{NO}_{3}$ is converted to $\mathrm{N}_{2}$, hence this process is also denoted denitrification.

${ }^{8}$ Anaerobic $=$ requires the absence of oxygen and nitrate oxides; $S_{\mathrm{O}_{2}} \approx 0, S_{\mathrm{NO}_{3}} \approx 0$.
} 
$\left(\mathrm{K}_{0.33} \mathrm{Mg}_{0.33} \mathrm{PO}_{3}\right)_{n}$ in stoichiometric computations). The phosphorous contents of $X_{\mathrm{PHA}}$ and $X_{\mathrm{PP}}$ are not included in $X_{\mathrm{PAO}}$.

Finally, since phosphorous removal and precipitation introduces mineral fractions in the wastewater which are not included in the other components, a total suspended solids $X_{\mathrm{TSS}}$ is introduced.

\subsection{Wastewater processes}

The overall chemical process taking place in BWWT can be described as (Metcalf \& Eddy 2003):

$v_{1}($ organic material $)+v_{2} \mathrm{O}_{2}+v_{3} \mathrm{NH}_{3}+v_{4} \mathrm{PO}_{4}^{3-} \rightarrow v_{5}($ new cells $)+v_{6} \mathrm{CO}_{2}+v_{7} \mathrm{H}_{2} \mathrm{O}$,

where the $v_{j}$-s are stoichiometric coefficients and it is assumed that microorganism enzymes have a catalytic effect on the reaction, but also that microorganisms grow and breed (new cells). Essentially, organic material is transformed into new cells under the consumption of oxygen, ammonia, and phosphorous components, producing water, $\mathrm{CO}_{2}$, and new cells which flocculate and may sediment.

In a realistic mathematical model, a more detailed set of stoichiometric reactions must be incorporated. In the following description of the chemical processes taking place between substrate and microorganisms, coefficients $v_{j}, i_{j}, f_{j}$, and $Y_{j}$ constitute a parameterization of the stoichiometric coefficients (Henze et al. 1999); in all chemical reactions below, the parameterized stoichiometric coefficients are positive. Four groups of chemical processes are introduced: Hydrolysis processes (decomposition of slowly biodegradable organic substrate $X_{\mathrm{S}}$ ), Processes of facultative heterotrophic organisms ${ }^{9}$ (metabolism and breeding of $X_{\mathrm{H}}$, etc.), Processes of phosphorous-accumulating organisms (involving $X_{\mathrm{PAO}}$ and their cell-internal storages $X_{\mathrm{PHA}}$ and $X_{\mathrm{PP}}$ ), and Nitrification processes (involving $S_{\mathrm{NH}_{4}}, S_{\mathrm{NO}_{3}}$, and $S_{\mathrm{N}_{2}}$ ).

A final comment before going into the some details of the chemical processes in wastewater treatment: there are some inconsistencies in Henze et al. (1999). As an example, in their Table 3, the stoichiometric coefficients for "Aerobic growth on $S_{\mathrm{F}}$ " are given, involving a number of species. However, in their summary Table 11, more species appear to be involved in the "reaction" than in their Table 3. The same is true for other processes. In the discussion below, the species that are discussed in their section "Biological processes, stoichiometry and kinetics" are underlined in the reaction schemes (the main species), while the additional species (as of their Table 11) are kept without underlining, and are given an arbitrary stoichiometric coefficient $v_{j}$.

Hydrolysis: The following hydrolysis (decomposition) reactions take place on slowly biodegradable substrate $X_{\mathrm{S}}$, catalyzed by enzymes in three classes of microorganisms, and split into soluble unbiodegradable $S_{\mathrm{I}}$ and fermentable $S_{\mathrm{F}}$ matter with a splitting factor $f_{\mathrm{SI}}$ :

$$
\underline{X_{\mathrm{S}}+\left(-v_{j, \mathrm{TSS}}\right) X_{\mathrm{TSS}}} \rightarrow \underline{f_{\mathrm{SI}} S_{\mathrm{I}}+\left(1-f_{\mathrm{SI}}\right) S_{\mathrm{F}}+v_{j, \mathrm{NH}_{4}} S_{\mathrm{NH}_{4}}+v_{j, \mathrm{PO}_{4}} S_{\mathrm{PO}_{4}}+v_{j, \mathrm{ALK}} S_{\mathrm{ALK}}}
$$

where, $j=1$ is aerobic hydrolysis $\left(S_{\mathrm{O}_{2}}>0\right), j=2$ is anoxic hydrolysis $\left(S_{\mathrm{O}_{2}} \approx 0\right.$, $\left.S_{\mathrm{NO}_{3}}>0\right)$, and $j=3$ is anaerobic hydrolysis $\left(S_{\mathrm{O}_{2}} \approx 0, S_{\mathrm{NO}_{3}} \approx 0\right)$.

${ }^{9}$ Facultative $=$ exhibiting a certain behavior under some environmental conditions, but not under other conditions. 
Facultative heterotrophic organisms: Facultative heterotrophic organisms metabolize and breed on substrates. Six chemical processes are considered. In oxygen based aerobic growth of $X_{\mathrm{H}}$ on fermentable substrate $S_{\mathrm{F}}$ :

$\frac{1}{Y_{\mathrm{H}}} S_{\mathrm{F}}+\left(\frac{1}{Y_{\mathrm{H}}}-1\right) S_{\mathrm{O}_{2}}+v_{4, \mathrm{NH}_{4}} S_{\mathrm{NH}_{4}}+v_{4, \mathrm{PO}_{4}} S_{\mathrm{PO}_{4}}+v_{4, \mathrm{ALK}} S_{\mathrm{ALK}} \rightarrow \underline{X_{\mathrm{H}}}+v_{4}, \mathrm{TSS} X_{\mathrm{TSS}}$,

in oxygen based aerobic growth of $X_{\mathrm{H}}$ on fermentation products $S_{\mathrm{A}}$ :

$\frac{1}{Y_{\mathrm{H}}} S_{\mathrm{A}}+\left(\frac{1}{Y_{\mathrm{H}}}-1\right) S_{\mathrm{O}_{2}}+v_{5, \mathrm{NH}_{4}} S_{\mathrm{NH}_{4}}+v_{5, \mathrm{PO}_{4}} S_{\mathrm{PO}_{4}} \rightarrow \underline{X_{\mathrm{H}}}+v_{5, \mathrm{TSS}} X_{\mathrm{TSS}}+v_{5, \mathrm{ALK}} S_{\mathrm{ALK}}$,

in nitrate based anoxic growth of $X_{\mathrm{H}}$ on fermentable substrate $S_{\mathrm{F}}$ :

$\underline{\frac{1}{Y_{\mathrm{H}}} S_{\mathrm{F}}+\frac{1-Y_{\mathrm{H}}}{2.86 Y_{\mathrm{H}}} S_{\mathrm{NO}_{3}}+v_{6, \mathrm{NH}_{4}} S_{\mathrm{NH}_{4}}+v_{6, \mathrm{PO}_{4}} S_{\mathrm{PO}_{4}} \rightarrow \underline{X_{\mathrm{H}}+\frac{1-Y_{\mathrm{H}}}{2.86 Y_{\mathrm{H}}} S_{\mathrm{N}_{2}}}}$

$+v_{6, \mathrm{TSS}} X_{\mathrm{TSS}}+v_{6, \mathrm{ALK}} S_{\mathrm{ALK}}$

while in nitrate based anoxic growth of $X_{\mathrm{H}}$ on fermentation products $S_{\mathrm{A}}$ :

$\frac{1}{Y_{\mathrm{H}}} S_{\mathrm{A}}+\frac{1-Y_{\mathrm{H}}}{2.86 Y_{\mathrm{H}}} S_{\mathrm{NO}_{3}}+v_{7, \mathrm{NH}_{4}} S_{\mathrm{NH}_{4}}+v_{7, \mathrm{PO}_{4}} S_{\mathrm{PO}_{4}} \rightarrow X_{\mathrm{H}}+\frac{1-Y_{\mathrm{H}}}{2.86 Y_{\mathrm{H}}} S_{\mathrm{N}_{2}}$

$+v_{7, \mathrm{TSS}} X_{\mathrm{TSS}}+v_{7, \mathrm{ALK}} S_{\mathrm{ALK}}$,

Under anaerobic conditions $\left(S_{\mathrm{O}_{2}} \approx 0, S_{\mathrm{NO}_{3}} \approx 0\right)$, the heterotrophic microorganisms do not grow. Instead $X_{\mathrm{H}}$ catalyze fermentation of $S_{\mathrm{F}}$ leading to fermentation products $S_{\mathrm{A}}$ :

$$
\underline{S_{\mathrm{F}}}+v_{8, \mathrm{ALK}} S_{\mathrm{ALK}} \rightarrow \underline{S \mathrm{~A}}+v_{8, \mathrm{NH}_{4}} S_{\mathrm{NH}_{4}}+v_{8, \mathrm{PO}_{4}} S_{\mathrm{PO}_{4}} .
$$

Finally, lysis reactions (decomposition/decay) occur in the heterotrophic organisms, where essentially $X_{\mathrm{H}}$ is split into unbiodegradable matter $X_{\mathrm{I}}$ and slowly biodegradable substrate $X_{\mathrm{S}}$ with a certain splitting factor $f_{\mathrm{XI}}$ :

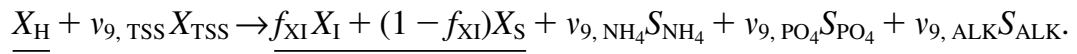

Phosphorous-accumulating organisms: Organisms $X_{\mathrm{PAO}}$ have the potential to accumulate phosphorous in the form of poly-phosphate $X_{\mathrm{PP}}$. Eight chemical processes are considered. Poly-hydroxy-alkanoates $X_{\mathrm{PHA}}$ are created from fermentation products $S_{\mathrm{A}}$ and stored $^{10}$ :

$$
\underline{S_{\mathrm{A}}+Y_{\mathrm{PO}_{4}} X_{\mathrm{PP}}+v_{10, \mathrm{TSS}} X_{\mathrm{TSS}}} \rightarrow \underline{X_{\mathrm{PHA}}}+Y_{\mathrm{PO}_{4} S_{\mathrm{PO}_{4}}+v_{10, \mathrm{ALK}} S_{\mathrm{ALK}} .}
$$

Poly-phosphates $X_{\mathrm{PP}}$ grow aerobically and anoxically:

$$
\underline{S_{\mathrm{PO}_{4}}+Y_{\mathrm{PHA}} S_{\mathrm{O}_{2}}+Y_{\mathrm{PHA}} X_{\mathrm{PHA}}} \rightarrow X_{\mathrm{PP}}+v_{11, \mathrm{TSS}} X_{\mathrm{TSS}}+v_{11, \mathrm{ALK}} S_{\mathrm{ALK}},
$$

$\underline{S_{\mathrm{PO}_{4}}+\left(-v_{12}, \mathrm{NO}_{3}\right) S_{\mathrm{NO}_{3}}+Y_{\mathrm{PHA}} X_{\mathrm{PHA}}} \rightarrow \underline{X_{\mathrm{PP}}+\left(-v_{12, \mathrm{NO}_{3}}\right) S_{\mathrm{N}_{2}}}$

$+v_{12, \mathrm{TSS}} X_{\mathrm{TSS}}+v_{12, \mathrm{ALK}} S_{\mathrm{ALK}}$.

${ }^{10}$ In Table 4 of Henze et al. (1999), $X_{\mathrm{PHA}}$ is assigned the stoichiometric coefficient 0 by misprint. 
Likewise, the organisms $X_{\mathrm{PAO}}$ grow aerobically and anoxically:

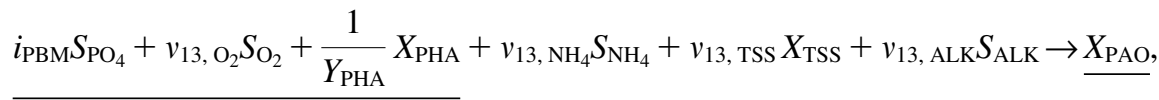

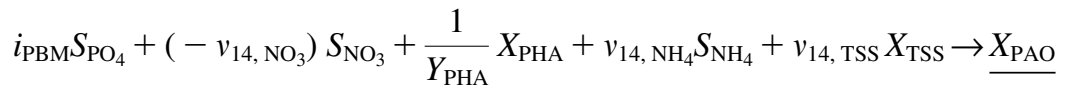

$+\left(-v_{14, \mathrm{NO}_{3}}\right) S_{\mathrm{N}_{2}}+v_{14, \mathrm{ALK}} S_{\mathrm{ALK}}$

Finally, each of the phosphorous particles $X_{\mathrm{PAO}}, X_{\mathrm{PP}}$, and $X_{\mathrm{PHA}}$ are prone to lysis (decomposition):

$$
\begin{aligned}
& \underline{X_{\mathrm{PAO}}}+v_{15, \mathrm{TSS}} X_{\mathrm{TSS}} \rightarrow \underline{f_{\mathrm{XI}} X_{\mathrm{I}}+\left(1-f_{\mathrm{XI}}\right) X_{\mathrm{S}}+v_{15, \mathrm{PO}_{4}} S_{\mathrm{PO}_{4}}}+v_{15, \mathrm{NH}_{4} S_{\mathrm{NH}_{4}}+v_{15, \mathrm{ALK}} S_{\mathrm{ALK}}}, \\
& \underline{X_{\mathrm{PP}}}+v_{16, \mathrm{TSS}} X_{\mathrm{TSS}}+v_{16, \mathrm{ALK}} S_{\mathrm{ALK}} \rightarrow \underline{S_{\mathrm{PO}_{4}}} \\
& \underline{X_{\mathrm{PHA}}}+v_{17, \mathrm{TSS}} X_{\mathrm{TSS}}+v_{17, \mathrm{ALK}} S_{\mathrm{ALK}} \rightarrow \underline{S_{\mathrm{A}}} .
\end{aligned}
$$

Nitrifying organisms: In anoxic processes, autotrophic organisms utilize oxygen from nitrate ions $\left(\mathrm{NO}_{3}^{-}\right.$, denitrification), producing nitrogen $\mathrm{N}_{2}$. This nitrate is produced in the nitrification process, which is a two step process, typically:

$$
\begin{aligned}
2 \mathrm{NH}_{4}^{+}+3 \mathrm{O}_{2} & \rightarrow 2 \mathrm{NO}_{2}^{-}+4 \mathrm{H}^{+}+2 \mathrm{H}_{2} \mathrm{O} \\
2 \mathrm{NO}_{2}^{-}+\mathrm{O}_{2} & \rightarrow 2 \mathrm{NO}_{3}^{-} .
\end{aligned}
$$

Since the reaction of nitrite $\left(\mathrm{NO}_{2}^{-}\right)$into nitrate is very fast, nitrite will hardly be present, and it is convenient to operate with a simplified overall stoichiometric reaction:

$$
\mathrm{NH}_{4}^{+}+2 \mathrm{O}_{2} \rightarrow \mathrm{NO}_{3}^{-}+2 \mathrm{H}^{+}+\mathrm{H}_{2} \mathrm{O}
$$

Utilizing the ASM notation, we write this nitrification process as:

$$
\begin{aligned}
& \frac{\left(-v_{18, \mathrm{NH}_{4}}\right) S_{\mathrm{NH}_{4}}+\frac{4.57-Y_{\mathrm{A}}}{Y_{\mathrm{A}}} S_{\mathrm{O}_{2}}+i_{\mathrm{PBM}} S_{\mathrm{PO}_{4}}}{}+v_{18, \mathrm{ALK}} S_{\mathrm{ALK}} \rightarrow \\
& \frac{1}{Y_{\mathrm{A}}} S_{\mathrm{NO}_{3}}+X_{\mathrm{AUT}}+v_{18, \mathrm{TSS}} X_{\mathrm{TSS}}
\end{aligned}
$$

Also, the autotrophic organisms are prone to lysis:

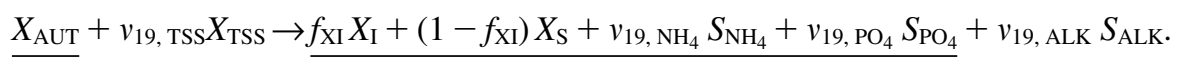

In Henze et al. (1999), two processes for chemical precipitation of phosphates are included; we have neglected these processes in our model.

\subsection{Wastewater kinetics and dynamic models}

A stoichiometric equation for a chemical process is typically written as

$$
v_{A} A+v_{B} B \rightarrow v_{C} C+v_{D} D+v_{E} E
$$


If $A, B, C, D$, and $E$ are unrelated, we can not say much more about the stoichiometric coefficients $v_{j}$. However, if $A=\mathrm{NH}_{4}^{+}, B=\mathrm{O}_{2}, C=\mathrm{NO}_{3}^{-}, D=\mathrm{H}^{+}$, and $E=\mathrm{H}_{2} \mathrm{O},{ }^{11}$ conservation of atoms and charge dictates that $v_{A}=k, v_{B}=2 k, v_{C}=k, v_{D}=2 k$, and $v_{E}=k$, where $k$ conveniently can be chosen as 1 (Smith \& Missen 1991). We can use conservation of atoms and charge to relate the stoichiometric coefficientsof the ASM2d, hence parametric description of the coefficients $v_{i}\left(Y_{j}, f_{k}, i_{l}\right)$ can be found; see Henze et al. (1999) for typical parameters.

With known stoichiometric equations and coefficients, kinetic rate expressions $\rho_{j}\left[\frac{\text { matter }}{\text { volume } \text { time }}\right]$ are needed, one for each chemical process. For all rates $\rho_{j}$, the rate is proportional to the concentration of the involved microorganism:

1. Hydrolysis and heterotroph growth depend on the presence of heterotroph organisms $X_{\mathrm{H}}$, hence $\forall j \in\{1, \ldots, 9\}: \rho_{j} \propto X_{\mathrm{H}}$.

2. The processes depending on phosphorous-accumulating organisms $X_{\mathrm{PAO}}$ have rates $\forall j \in\{10, \ldots, 17\}: \rho_{j} \propto X_{\mathrm{PAO}}$.

3. Processes governed by nitrifying organisms (autotroph organisms) have rates $\forall j \in\{18,19\}: \rho_{j} \propto X_{\mathrm{AUT}}$.

Next, the hydrolysis process rates depend on the simultaneous presence of substrate $X_{\mathrm{S}}$ and microorganism $X_{\mathrm{H}}$; these rates depend on how well $X_{\mathrm{S}}$ covers the surface of $X_{\mathrm{H}}$, hence

$$
\forall j \in\{1,2,3\}: \rho_{j} \propto \frac{X_{\mathrm{S}} / X_{\mathrm{H}}}{K_{\mathrm{X}}+X_{\mathrm{S}} / X_{\mathrm{H}}} \rightarrow\left\{\begin{array}{ll}
0, & \frac{X_{\mathrm{S}}}{X_{\mathrm{H}}} \rightarrow 0 \\
1, & \frac{X_{\mathrm{S}}}{X_{\mathrm{H}}} \rightarrow \infty
\end{array} .\right.
$$

Similar rate expressions are used for other processes where microorganisms catalyze transformation of material, e.g. for storage of $X_{\mathrm{PHA}}$ (process 10) and aerobic storage of $X_{\mathrm{PHA}}$ and $X_{\mathrm{PP}}$ (processes 11 and 13).

Finally, some switching functions are introduced in order to model how the rates depend on the presence or absence of substrates. As an example, aerobic processes are assumed to depend on the presence of oxygen, hence the rates are made proportional to

$$
\forall j \in\{1,4,5,11,13,18\}: \rho_{j} \propto \frac{S_{\mathrm{O}_{2}}}{K_{\mathrm{O}_{2}}+S_{\mathrm{O}_{2}}} \rightarrow\left\{\begin{array}{ll}
0, & S_{\mathrm{O}_{2}} \rightarrow 0 \\
1, & S_{\mathrm{O}_{2}} \rightarrow \infty
\end{array} .\right.
$$

On the other hand, for anoxic and anaerobic processes that depend on the absence of oxygen, the rates are made proportional to either

$$
\forall j \in\{2,3,6,7,8\}: \rho_{j} \propto \frac{K_{\mathrm{O}_{2}}}{K_{\mathrm{O}_{2}}+S_{\mathrm{O}_{2}}} \rightarrow \begin{cases}1, & S_{\mathrm{O}_{2}} \rightarrow 0 \\ 0, & S_{\mathrm{O}_{2}} \rightarrow \infty\end{cases}
$$

or

$$
\forall j \in\{12,14\}: \rho_{j} \propto \frac{K_{\mathrm{O}_{2}}}{S_{\mathrm{O}_{2}}} \rightarrow \rightarrow \begin{cases}\infty, & S_{\mathrm{O}_{2}} \rightarrow 0 \\ 0, & S_{\mathrm{O}_{2}} \rightarrow \infty\end{cases}
$$

Similar switching functions are used to model the required presence or absence of nitrate $\mathrm{S}_{\mathrm{NO}_{3}}$. 
The full set of process rate equations $\rho_{j}$ are given in Table 7 of Henze et al. (1999). The final step in the modeling phase is to formulate specie balances, which typically have the form

$$
\frac{d}{d t} m_{j}=\dot{m}_{\mathrm{i}, j}-\dot{m}_{\mathrm{e}, j}+\dot{m}_{\mathrm{g}, j}
$$

where $m_{j}$ is the accumulated mass of specie $j$ (e.g. $S_{\mathrm{F}}, X_{\mathrm{H}}$, etc.), $\dot{m}_{\mathrm{i}, j}$ is the mass influx of specie $j$ into a system, $\dot{m}_{\mathrm{e}, j}$ is the mass efflux of specie $j$ out of a system, and $\dot{m}_{\mathrm{g}, j}$ is the mass of specie $j$ generated in a chemical reaction per time unit. For a well mixed system of volume $V$,

$$
\dot{m}_{\mathrm{g}, j}=V \cdot \sum_{k=1}^{K} v_{k, j} \rho_{k},
$$

where $K$ chemical processes are taking place; $K=19$ in our slightly simplified ASM2d description. In our description, we use either 18 or 17 species, depending on whether we include $S_{\mathrm{S}}=S_{\mathrm{F}}+S_{\mathrm{A}}$ or not. Thus, we will have 18 (or 17) differential equation for mass accumulation for each well mixed system volume in our biological wastewater treatment plant.

\section{Parameter Identifiability}

\subsection{System, model, and parameter}

For a system $\mathcal{S}$, assume that we have developed a model structure $\mathcal{M}$ with parameter $\theta \in \mathbb{R}^{n}$; the corresponding model is denoted $\mathcal{M}(\theta)$. The (parametric) model may be based on a mechanistic understanding of the system $\mathcal{S}$, or be a generic mathematical mapping. By varying $\theta$ over a feasible set $\Theta$, we get a set of models $\mathcal{M}(\Theta)$, i.e. a model structure $\mathcal{M}$. In reality, the model behavior depends on the experimental conditions $\mathcal{K}$; $\mathcal{K}$ is a description of how an experiment has been carried out, including a sequence of inputs $u_{t} \in \mathbb{R}^{n_{u}}, t \in\{1, \ldots, T\}$ to, and outputs $y_{t} \in \mathbb{R}^{n_{y}}, t \in\{1, \ldots, T\}$ from the real system $\mathcal{S}$. With a given model $\mathcal{M}(\theta)$ and experimental conditions $\mathcal{K}$, we can compute a model output $y_{t}^{m}(\mathcal{M}(\theta), \mathcal{K})$. We will simplify the notation for the model output to $y_{t}^{m}(\theta)$.

With available model structure $\mathcal{M}(\Theta)$ and experimental conditions $\mathcal{K}$, we can attempt to find a specific parameter $\hat{\theta}$ which is such that the model output $y_{t}^{m}(\hat{\theta})$ is close to the system output $y_{t}$ in some sense. To achieve this, we can choose from a set of identification methods $\mathcal{I}$; the estimate $\hat{\theta}$ will also depend on the chosen identification method $\mathcal{I}$. We will simplify the notation and write $y_{t}^{m}(\hat{\theta})$ as $\hat{y}_{t}$.

Usually, it is assumed that the real system $\mathcal{S}$ is an element of the model structure $\mathcal{M}(\Theta)$ and has a true parameter $\theta^{*}$ such that $\mathcal{S}$ is (input-output) identical to $\mathcal{M}\left(\theta^{*}\right)$. It is thus of interest to study whether and to what degree it is possible to find the true system parameters $\theta^{*}$ from the given model structure $\mathcal{M}(\Theta)$, experimental conditions $\mathcal{K}$, and identification method $\mathcal{I}$. We will denote this study an experimental parameter identifiability study, since the result depends on the experimental conditions $\mathcal{K}$ (and to some degree on $\mathcal{I}$ ). Another important study could be whether and to what degree $\theta^{*}$ can be found from $\mathcal{M}(\Theta)$ if we allow any (conceivable) experimental conditions $\mathcal{K}$; this we will denote a theoretical parameter identifiability study.

Clearly, the set of experimentally identifiable parameters will be a subset of the set of theoretically identifiable parameters. Examples of methods for studying theoretical parameter identifiability, are given in e.g. Pohjanpalo (1978) and Holmberg (1982), and in Ljung \& Glad (1994); see also Walter \& Pronzato (1997). However, for many 
industrial processes it will be difficult to get accept for carrying out active experiments, and it may be necessary to rely on the natural perturbation in the system. Thus, we may not be able to identify all the theoretically identifiable parameters in practice, and the experimental identifiability may give a better measure of the parameters which can be found.

After a set of identifiable parameters have been found, these can be estimated using some identification technique $\mathcal{I}$. Then, the statistical quality of the model parameters $\hat{\theta}$ and prediction model $\hat{y}_{t}$ should be studied.

Parameter identifiability and parameter estimation in mechanistic models has been widely studied in engineering (Bard 1974), (Beck \& Arnold 1977), (Walter \& Pronzato 1997), (Rawlings \& Ekerdt 2002), (Ljung 1999), (Söderström \& Stoica 1989). The systematic approach described in Brun, Reichert \& Künsch (2001) seems well suited to Biological Waste Water Models, since it handles large simulation models, and provides identifiability diagnosis for parameter subsets. In the sequel, some background to the method of Brun et al. (2001) is given.

\subsection{Practical identifiability analysis}

We assume that the output $y_{t} \in \mathbb{R}^{n_{y} \times 1}$ and the model output $y_{t}^{m}(\theta \mid \mathcal{K})$ are related by $y_{t}=y_{t}^{m}(\theta \mid \mathcal{K})+e_{t}$, where $e_{t}$ describes model error/uncertainty. Furthermore, both $y_{t}$ and $u_{t}$ are available for $t \in\{1, \ldots, T\}$ (experimental conditions $\mathcal{K}$ ) and we introduce the notation $y=y^{m}(\theta \mid \mathcal{K})+e$ to describe all the resulting equations; $y, y^{m}, e \in \mathbb{R}^{n_{y}} \cdot T \times 1$. With a perfect model structure and the correct parameter vector $\theta^{*}$, the error $e$ will be $e \equiv 0$ and $y \equiv y^{m}\left(\theta^{*} \mid \mathcal{K}\right)$. In the real world, $e$ is unknown. A possible strategy is to set $e=0$, and hope for a solution $\theta$ such that $y=y^{m}(\theta \mid \mathcal{K})$. We base the analysis on a linearized approximation of this equation, giving $T \cdot n_{y}$ linear equations in $n_{\theta}$ unknowns:

$$
S \cdot \Delta \hat{\theta}=\Delta y,
$$

where $\Delta y \triangleq y-y^{m}\left(\theta^{i} \mid \mathcal{K}\right), \Delta \hat{\theta} \triangleq \hat{\theta}-\theta^{i},\left.S \triangleq \frac{\partial y^{m}}{\partial \theta}\right|_{\theta=\theta^{i} .} S$ is denoted the model sensitivity. If no solutionexists, it is common to project $\Delta y$ on the column space of $S, \Delta y_{\mathcal{R}(S)}$, and solve $S \cdot \Delta \hat{\theta}=\Delta y_{\mathcal{R}(S)}$-this equation always has a solution, which coincides with the solution of the corresponding normal equation from using the least squares method:

$$
S^{T} S \cdot \Delta \hat{\theta}=S^{T} \cdot \Delta y .
$$

The chosen method for analyzing the identifiability of parameters, is based on Brun et al. (2001), see also Brun et al. (2002), Dueñas Díez, Fjeld, Andersen \& Lie (2006). This method is rooted in the linear equation of either equation 1, or 2. Essentially, these equations have a solution if the sensitivity matrix $S$ has full rank. Clearly, if the $j$ th column $s_{j}$ of matrix $S$ is a zero vector, then matrix $S$ exhibits rank loss and parameter $\theta_{j}$ can not be found. Similarly, if $\left\|s_{j}\right\|$ is "small", we may expect problems in finding $\theta_{j}$. However, even when all columns of $S$ are significantly different from zero vectors, we may have problems in finding $\theta$ if some column is linearly dependent on the other columns. This linear dependence is termed collinearity. Two possible measures of collinearity are the condition number $\kappa_{j}$, and the smallest singular value $\sigma_{\min }(S)$.

Scaling of outputs and parameters is essential, and it is recommended to use dimensionless quantities $\tilde{y}_{t, i}=y_{t, i} / y_{i}^{\circ}$ and $\tilde{\theta}_{i}=\theta_{i} / \theta_{i}^{\circ}$. In the sequel, it is assumed that such a scaling has been introduced as part of the preprocessing of the data. 
In their method, Brun et al. (2001) consider the sensitivity measure $\delta_{j}^{\text {msqr }}$ defined as

$$
\delta_{j}^{\mathrm{msqr}} \triangleq \frac{1}{\sqrt{n_{y} T}}\left\|s_{j}\right\|_{2} .
$$

Their strategy is then to sort the various parameters according to the value of $\delta_{j}^{\mathrm{msqr}}$. If there is a marked drop in the value of $\delta_{j}^{\text {msqr }}$ for some $j$, then they propose to assume that those parameters with the smallest sensitivity measure can not be found; hence these parameters are removed from the set of tunable parameters. It should be noted, however, that if the sensitivity measure decreases without marked jumps, and if it is within a couple of decades from the largest value, it may be unjustified to remove a parameter. In typical applications, a set of 10-20 parameters are often retained in the parameter set after the sensitivity screening described above.

The chosen measure of collinearity index $\gamma(\theta)$ is

$$
\gamma(\theta) \triangleq \frac{1}{\sigma_{\min }(\tilde{S})}=\frac{1}{\sqrt{\sigma_{\min }\left(\tilde{S}^{T} \tilde{S}\right)}},
$$

where $\tilde{S}$ is a slightly modified sensitivity matrix where the columns have been normalized to unit norm. It should be noted that this measure is not necessarily a good measure of collinearity, see Stewart (1987) and Belsley (1991).

Collinearity is a measure of how combinations of parameters interact, thus they propose to consider all possible subsets $P$ of parameters of $\theta$, and compute the collinearity index for all these possible subsets. Let $\theta_{p}$ be one of these subsets, thus $\gamma\left(\theta_{p}\right)$ is to be computed for all possible $p \in \mathcal{P}$. It follows from basic combinatorics that the number of possible subsets with at least 2 parameters and at most $n_{\theta}$ parameters is $\# \gamma\left(\theta_{P}\right)=\sum_{i=2}^{n_{\theta}}\left(\begin{array}{c}n_{\theta} \\ i\end{array}\right)$; with $n_{\theta}=10, \# \gamma\left(\theta_{T}\right) \approx 10^{3}$, and with $n_{\theta}=20, \# \gamma\left(\theta_{T}\right) \approx 10^{6}$.

In their papers, Brun et al. propose that when $\gamma\left(\theta_{p}\right)>10$, parameter set $p$ should be considered collinear. Thus, all parameter sets $p$ for which $\gamma\left(\theta_{p}\right) \leq 10$ should be considered for further study, and parameter estimates for these sets $p$ should be computed. The final choice of parameters is then based on a statistical analysis of theparameters for which $\gamma\left(\theta_{p}\right)$ is approximately less than 10 .

\section{The Duvbacken Plant Model}

\subsection{Process flow description}

Wastewater first undergoes pretreatment involving removal of coarse particles and primary sedimentation of particles. Referring to Figure 1, the pretreated wastewater together with sludge return enters the anaerobe reactor step consisting of three parallel lines, each line consisting of two separate volumes in series, i.e. the anaerobe reactor step consists of a total of 6 separate volumes-each of which is supplied with mixers. The main purpose of the anaerobic reactor step, is to make conditions suitable for biological phosphorous removal.

Aerobe reactor step 1 has the same physical configuration as has the anaerobe reactor step, that is, three parallel lines, each line consisting of two separate volumes. All six volumes are aerated, either by continuous aeration or by intermittent aeration. The air is supplied from three blowers, serving a common air line distributing air to each of the six volumes - which in principle can be controlled independently-where the air enters each volume through about 140 aeration membrane dishes. The outlets from the three parallel lines converge into a common effluent line. 


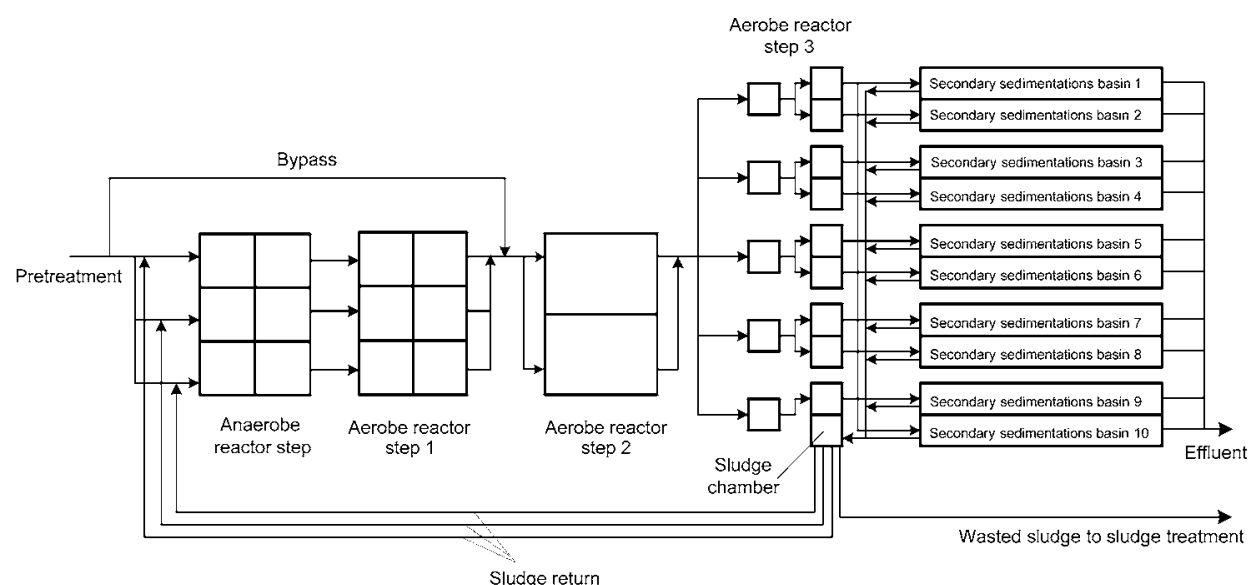

Figure 1. Flowsheet of the Duvbacken Waste Water Treatment Plant.

As can be seen from Figure 1, it is also possible to bypass a certain portion of the pretreated wastewater directly to aerobe reactor step 2 . The reason for this is that effluent from the pre-treatment is rich in readily biodegradable organic matter. Hence, as to enhance denitrification in aerobe reactor step 2 (if biological nitrogen removal is the goal), bypass of the pretreated wastewater directly to reactor step 2 might be a useful strategy. Aerobe reactor step 2 consists of two rectangular and parallel basins. Each basin is aerated, either continuously or intermittently, and the air is supplied using four ejectors in each basin, distributed equally along the length of the basins.

From aerobe reactor step 2, the outlet lines converge into one effluent line which leads to aerobe reactor step 3. Aerobe reactor step 3 consists of fourteen small volumes, configured as shown in Figure 1. Each volume is aerated, either continuously or intermittently. The air is supplied from blowers, and the air is dispersed in each volume through aeration dishes.

From aerobe reactor step 3, the wastewater flows into ten parallel secondary sedimentation basins. The sludge from these basins, is pumped into a sludge chamber. The sludge chamber has two main purposes. Firstly, sludge is returned back to the inlet of the anaerobe reactor step-one sludge return line for each of the three parallel lines. Secondly, sludge is wasted and pumped to the sludge treatment which involves addition of polymer, thickening etc. The reject water from the thickening process is returned back to the plant.

\subsection{Flow descriptions and sensors}

The component concentrations in the influent to the plant is typically characterized as follows, Table 2. With reference to Table 2, the following comments should be made:

- The pretreatment reduces the total phosphorous content considerably due to sludge removal from the primary sedimentation basins.

- The pre-treatment reduces the unfiltered biological oxygen demand with 50 . The average volumetric influent flow rate is $1400-1500 \mathrm{~m}^{3} / \mathrm{h}$.

The plant has to obey the following governmental effluent requirements:

- $\mathrm{BOD}_{7}<10 \mathrm{mg}=1$.

- $\mathrm{P}_{\text {tot }}<0.5 \mathrm{mg}=1$. 
Table 2. Description of typical influent concentrations to the Duvbacken Waste Water Treatment Plant.

\begin{tabular}{|c|c|c|c|c|c|c|}
\hline \multirow[b]{2}{*}{ Parameters } & \multirow[b]{2}{*}{ Description } & \multirow[b]{2}{*}{ Units } & \multicolumn{2}{|c|}{ Influent to plant } & \multicolumn{2}{|c|}{ After pretreatment } \\
\hline & & & Unfiltered & Filtered & Unfiltered & Filtered \\
\hline $\mathrm{P}_{\text {tot }}$ & Total phosphorous & $\mathrm{mg} / \mathrm{l}$ & 6.0 & 3.2 & 3.4 & 1.2 \\
\hline $\mathrm{PO}_{4}-\mathrm{P}$ & Phosphate phosphorous & $\mathrm{mg} / \mathrm{l}$ & & 3.4 & & 2.0 \\
\hline $\mathrm{NO}_{3}-\mathrm{N}$ & Nitrate nitrogen & $\mathrm{mg} / \mathrm{l}$ & & 1.0 & & 1.4 \\
\hline $\mathrm{NH}_{4}-\mathrm{N}$ & Ammonia nitrogen & $\mathrm{mg} / \mathrm{l}$ & & 27.2 & & \\
\hline $\mathrm{BOD}_{7}$ & Biological oxygen demand & $\mathrm{mg} / \mathrm{l}$ & 205 & 69 & 99 & 55 \\
\hline
\end{tabular}

Table 3. On-line sensors at the Duvbacken Waste Water Treatment Plant.

\begin{tabular}{|c|c|c|}
\hline Sensor type & Location & Sensors \\
\hline Dissolved oxygen $S_{\mathrm{O}_{2}}$ & $\begin{array}{l}\text { Aerobe reactor step } 1 \text {, one sensor in each parallel line. } \\
\text { Aerobe reactor step 2, two sensors in each basin. } \\
\text { Aerobe reactor step } 3,5 \text { sensors in } 5 \text { different volumes. }\end{array}$ & $\overline{y_{6}}$ \\
\hline Nitrate nitrogen $S_{\mathrm{NO}_{3}}$ & $\begin{array}{l}\text { Outlet of aerobe reactor step } 2 \text {, one sensor } \\
\text { Sludge chamber, one sensor. }\end{array}$ & $\begin{array}{l}y_{4} \\
y_{8}\end{array}$ \\
\hline Ammonia nitrogen $S_{\mathrm{NH}_{4}}$ & $\begin{array}{l}\text { Outlet of aerobe reactor } 1 \text {, one sensor. } \\
\text { Effluent of the plant, one sensor. }\end{array}$ & $\begin{array}{l}y_{1} \\
y_{9}\end{array}$ \\
\hline Phosphate phosphorous & Effluent of the plant, one sensor. & - \\
\hline Total phosphorous & Effluent of the plant, one sensor. & - \\
\hline Dissolved phosphorous $S_{\mathrm{PO}_{4}}$ & Outlet of aerobe reactor step 3 , one sensor. & $y_{10}$ \\
\hline Total solids $X_{\mathrm{A}}+X_{\mathrm{H}}+X_{\mathrm{PAO}}$ & $\begin{array}{l}\text { Outlet of aerobe reactor step } 1 \text {, one sensor. } \\
\text { Outlet of aerobe reactor step 2, one sensor. } \\
\text { Sludge chamber, one sensor } \\
\text { Effluent of plant, one sensor. }\end{array}$ & $\begin{array}{l}y_{3} \\
y_{7}\end{array}$ \\
\hline
\end{tabular}

As can be seen, there are currently no governmental requirements regarding the nitrogen contents in the effluent. Table 3 gives an overview of the available on-line sensors of the plant.

In addition, inorganic soluble phosphorous $S_{\mathrm{PO}_{4}}$ is measured by off-line laboratory analysis in aerobe reactors 1,2 , and $3 ; y_{2}, y_{5}$, and $y_{10}$, respectively.

\subsection{Model and model verification}

A dynamic model is developed, based on the standard kinetics of the ASM2d model (Henze et al. 1999), (Metcalf \& Eddy 2003), (Henze et al. 2000). In the dynamic model, each of the 4 steps in the plant (anaerobe, aerobe 1-3) is modeled as a perfectly stirred reactor. This leads to 17 states in each of 4 stirred volumes, i.e. 68 states. The model has a total of 45 parameters. The states of the model are grouped into the concentration of soluble species $S_{j}$ and particulate species $X_{j}$. The model has 18 potential input variables: total volumetric flow rate $q$ and the composition of the 17 species. In the model fitting, 

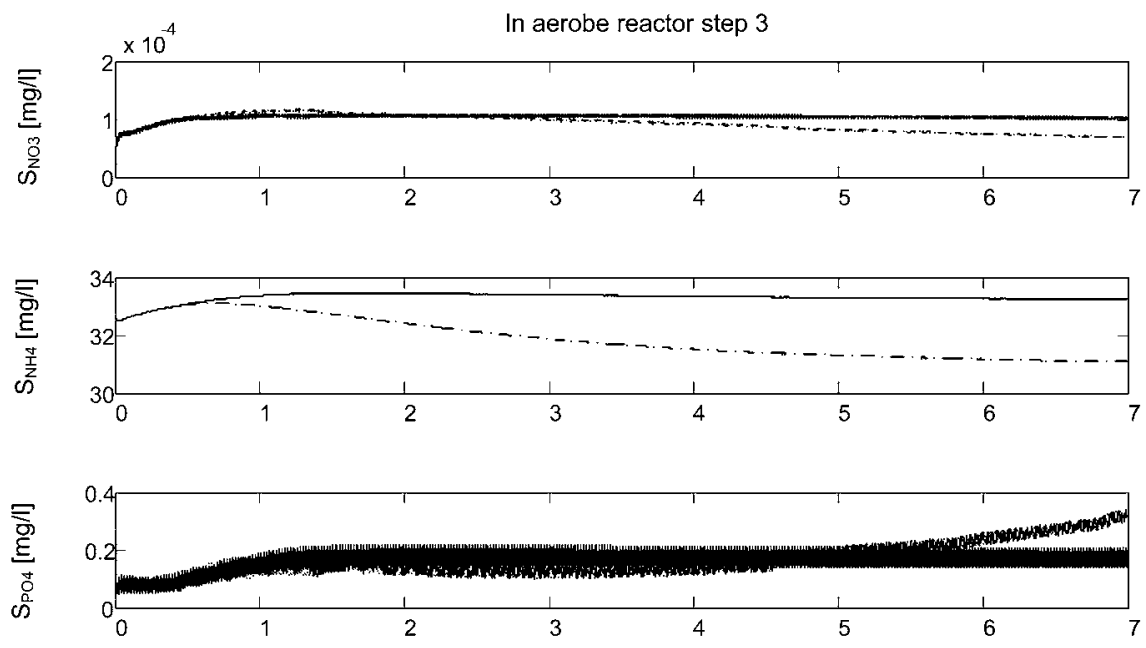

In sludge chamber

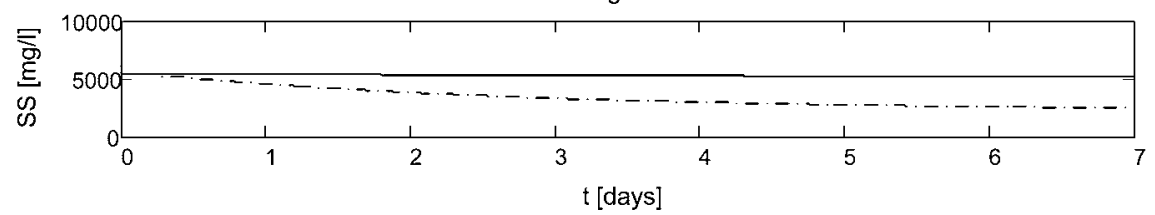

Figure 2. Simulated outputs $S_{\mathrm{NO}_{3}}, S_{\mathrm{NH}_{4}}, S_{\mathrm{PO}_{4}}$, and $\mathrm{SS}=X_{\mathrm{A}}+X_{\mathrm{H}}+X_{\mathrm{PAO}}$ over 7 days, with sludge ages $T_{\text {sludge }}=6 \mathrm{~d}$ (solid, black) and $T_{\text {sludge }}=2 \mathrm{~d}$ (dashed, blue).

10 measurements are available ${ }^{12}$. Examples of measurements are the concentration of suspended solids SS (i.e. the sum of the microorganisms), the concentration of soluble nitrates $S_{\mathrm{NO}_{3}}$, the concentration of soluble ammonium $S_{\mathrm{NH}_{4}}$, and the concentration of inorganic soluble phosphorous $S_{\mathrm{PO}_{4}}$. The model has been implemented in Matlab, and the calling syntax for running the simulation code and compute the outputs for the plant is

$$
[\mathrm{T}, \mathrm{Y}]=\mathrm{bioout}(\mathrm{s} 0, \mathrm{x} 0, \mathrm{q}, \mathrm{Sf}, \mathrm{Xf}, \mathrm{P} 0)
$$

Here, $\mathrm{s} 0$ and $\mathrm{x} 0$ are the initial state vectors, $\mathrm{q}$ is the feed flow rate, $\mathrm{Sf}$ and $\mathrm{Xf}$ are the feed concentrations of the state variables, and $\mathrm{PO}$ is the parameter vector. The response from the simulator is $\mathrm{T}$ which is a vector of time instants where the solution is available, and $\mathrm{Y}$ which is the matrix of output responses.

Before analyzing the identifiability and estimating parameters, it is a good idea to verify that the model gives reasonable responses (Olsson \& Newell 1999). Figure 2 depicts the concentrations of SS, $S_{\mathrm{NO}_{3}}, S_{\mathrm{NH}_{4}}$, and $S_{\mathrm{PO}_{4}}$ for two different sludge ages in the plant $-T_{\text {sludge }}=6 \mathrm{~d}$ and $T_{\text {sludge }}=2 \mathrm{~d},{ }^{13}$ during 7 days of operation. The results are reasonable:

- Figure 2 shows that the suspended solids concentration SS is reduced when the sludge age is reduced. This makes sense: less residence time means that less solids is precipitated.

\footnotetext{
${ }^{12}$ In this paper, all measurements were assumed to be available every minute.

${ }^{13}$ The sludge age is the residence time of the sludge in the reactor system.
} 
- To observe bio-N operation (nitrogen removal), in general the sludge age should be no less than $6 \mathrm{~d}$. To observe bio-P operation (phosphorous removal), the sludge age should be no less than $2 \mathrm{~d}$. Thus, with a sludge age of $6 \mathrm{~d}$ in Figure 2, the system should be on the verge of dropping out of bio- $\mathrm{N}$ operation, and with a sludge age of $2 \mathrm{~d}$, the system should be on the verge of dropping out of bio-P operation.

- Although bio- $\mathrm{N}$ operation may be obtained with a sludge age of $6 \mathrm{~d}$, in the scenarios simulated in Figure 2, the feed contains no autotrophic biomass $X_{\mathrm{AUT}}$, and the concentration of $X_{\mathrm{AUT}}$ in the effluent stays below $10^{-2} \mathrm{mg} / \mathrm{l}$ for the entire period of $7 \mathrm{~d}$ (not shown). To observe nitrification, $X_{\mathrm{AUT}}$ should have a value around, say, $300 \mathrm{mg} / \mathrm{l}$. Nitrification would then manifest itself in e.g. a marked decrease in dissolved ammonium $S_{\mathrm{NH}_{4}}$.

- Phosphorous removal is observed from e.g. the amount of dissolved phosphorous $S_{\mathrm{PO}_{4}}$. In Figure 2, the concentration of $S_{\mathrm{PO}_{4}}$ starts to increase (after $4 \mathrm{~d}$ ) in the case of the $2 \mathrm{~d}$ sludge age, while it stays low for the higher sludge age. This indicates bio-P operation at a sludge age of $6 \mathrm{~d}$, while the bio-P operation is failing at a sludge age of $2 \mathrm{~d}$.

There are some oscillations in some outputs in Figure 2-these are due to oscillating oxygen feed in the aerobe reactors.

\section{Identifiability Analysis for the Duvbacken Plant Model}

\subsection{Experimental conditions}

With our state space based model, we need a set of experimental input signals $u_{t}$ in order to carry out the identifiability analysis. Identifiability analysis as discussed in this paper, does not require knowledge of the experimental response $y_{t}$ from the real system. However, if we later want to estimate the parameters, then the system response is needed.

Here, we use a simulation model implemented in Matlab, with constant influent flow rate and compositions; the main transients in the system are due to initial values that are not at steady state, and an oscillating strategy for oxygen feed. Although the relatively small perturbation of the system is far from ideal for parameter identification, it will serve to illustrate the method for experimental parameter identifiability analysis.

\subsection{Model sensitivity}

The sensitivity was found by numerically perturbing the simulation model. First, a nominal output was computed, $y_{t}^{m}\left(\theta^{0}\right)$, and reshaped into $y^{m}\left(\theta^{0}\right) \in \mathbb{R}^{n_{y} T \times 1}$. Then the perturbed output $y_{t}^{m}\left(\theta^{0}+e_{j} \cdot \delta \theta_{j}\right)$ was computed and reshaped into $y^{m}\left(\theta^{0}+e_{j} \cdot \delta \theta_{j}\right)$; $e_{j}$ is column $j$ of the identity matrix $I \in \mathbb{R}^{n_{\theta} \times n_{\theta}}$. Then finally column $s_{j}$ of the sensitivity $S$ was computed as

$$
s_{j} \approx \frac{y^{m}\left(\theta^{0}+e_{j} \cdot \delta \theta_{j}\right)-y^{m}\left(\theta^{0}\right)}{\delta \theta_{j}} .
$$

The outputs and parameters were scaled according to recommendations in Brun et al. (2002). 
Table 4. Parameter importance ranking according to the sensitivity measures. Parameters labeled by an asterisk $(*)$ are considered identifiable from the available data.

\begin{tabular}{lllr}
$\delta_{j}^{\text {msqr }}$ & & \multicolumn{1}{c}{ Parameters } & Parameter \# \\
\hline 14.812 & $\delta_{\mathrm{PAO}}^{*}$ & Maximum growth rate of PAO & 30 \\
3.9867 & $q_{\mathrm{PP}}^{*}$ & Rate constant for storage of $X_{\mathrm{PP}}$ & 29 \\
3.2385 & $b_{\mathrm{PAO}}$ & Rate for Lysis of $X_{\mathrm{PAO}}$ & 32 \\
3.105 & $K_{\mathrm{X}}^{*}$ & Saturation coefficient for particulate COD & 8 \\
2.9954 & $K_{h}$ & Hydrolysis rate constant & 4 \\
2.9412 & $\mu_{\mathrm{H}}^{*}$ & Maximum growth rate on substrate & 16 \\
2.9324 & $q_{\mathrm{fe}}^{*}$ & Maximum rate for fermentation & 17 \\
2.5858 & $K_{\mathrm{A}, \mathrm{PAO}}^{*}$ & Saturation coefficient for acetate, $S_{\mathrm{A}}$ & 36 \\
2.2768 & $b_{\mathrm{PHA}}^{*}$ & Rate for Lysis of $X_{\mathrm{PHA}}$ & 33 \\
2.2196 & $q_{\mathrm{PHA}}^{*}$ & Rate constant for storage of $X_{\mathrm{PHA}}\left(\right.$ base $\left.X_{\mathrm{PP}}\right)$ & 28 \\
1.9906 & $K_{\mathrm{A}, \mathrm{HET}}$ & Saturation coefficient for growth on acetate $S_{\mathrm{A}}$ & 23 \\
1.9526 & $K_{\mathrm{PHA}}^{*}$ & Saturation coefficient for PHA & 44 \\
1.326 & $K_{\mathrm{O}_{2}, \mathrm{HYD}}$ & Saturation/inhibition coefficient for oxygen & 7 \\
1.2238 & $b_{\mathrm{HET}}^{*}$ & Rate for Lysis of $X_{\mathrm{H}}$ & 19 \\
1.1272 & $K_{\mathrm{O}_{2}, \mathrm{HET}}$ & Saturation/inhibition coefficient for oxygen & 6 \\
1.0583 & $\eta_{\mathrm{fe}}^{*}$ & Anoxic hydrolysis reduction factor & 34 \\
0.99162 & $K_{\mathrm{O} 2, \mathrm{PAO}}$ & Saturation/inhibition coefficient for oxygen & 21 \\
0.95771 & $K_{\mathrm{F}}$ & Saturation coefficient for growth on $S_{\mathrm{F}}$ & 38 \\
0.52842 & $K_{\mathrm{PS}, \mathrm{PAO}}^{*}$ & Saturation coefficient for phosphorus in storage of PP & 45 \\
0.46989 & $b_{\mathrm{PP}}^{*}$ & Rate for Lysis of $X_{\mathrm{PP}}$ & \\
\hline
\end{tabular}

\subsection{Sensitivity index}

The sensitivity measure $\delta_{j}^{\mathrm{msqr}}=\left\|s_{j}\right\|_{2} / \sqrt{n_{y} T}$ was computed. Out of the 45 original parameters, the 20 most sensitive parameters are shown in Table 4.

Although the decrease in $\delta_{j}^{\text {msqr }}$ is gradual, we thus make the deliberate choice of assuming that parameters $21-45$ are not identifiable. We assign the nominal parameter value to these parameters, and then continue with collinearity analysis to see which of the 20 parameters with highest sensitivity that we can estimate.

\subsection{Collinearity indices and identifiable parameters}

Similarly as to in Brun et al. (2002), the 20 parameters of interest from the parameter sensitivity ranking can be classified in different groups according to the biological processes to which they belong. The parameters are divided into 4 different groups according to the kind of biological processes they describe: 4 parameters are related to hydrolysis of particulate substrate, 6 parameters are related to heterotrophic organisms, 10 parameters are related to phosphorus-accumulating organisms, and none are related to autotrophic organisms. The distribution of the 20 most sensitive parameters is thus:

- Hydrolysis of particulate substrate: $K_{\mathrm{X}}, K_{\mathrm{h}}, K_{\mathrm{O}_{2}, \mathrm{HYD}}, \eta_{\mathrm{fe}}$

- Heterotrophic organisms: $\mu_{\mathrm{H}}, q_{\mathrm{fe}}, K_{\mathrm{A}, \mathrm{HET}}, b_{\mathrm{HET}}, K_{\mathrm{O}_{2}, \mathrm{HET}}, K_{\mathrm{F}}$

- Phosphorus-accumulating organisms: $\mu_{\mathrm{PAO}}, q_{\mathrm{PP}}, b_{\mathrm{PAO}}, K_{\mathrm{A}, \mathrm{PAO}}, b_{\mathrm{PHA}}, q_{\mathrm{PHA}}, K_{\mathrm{PHA}}$, $K_{\mathrm{O}_{2}, \mathrm{PAO}}, K_{\mathrm{PS}, \mathrm{PAO}}, b_{\mathrm{PP}}$

- Autotrophic organisms:-

Next, the collinearity index $\gamma\left(\theta_{p}\right)$ is calculated for all possible subsets of the top 20 parameters. Values for $\gamma\left(\theta_{p}\right)$ lie in the range $1-11$. This is almost within the range of 
$\gamma\left(\theta_{p}\right) \leq 10$ as proposed by Brun et al. (2002), so our parameters are hardly collinear. However, to illustrate the concept of collinearity, we instead consider parameters to be collinear if $\gamma\left(\theta_{p}\right)>5$. Here it is found that there are parameter subsets with up to 13 elements which fulfill $\gamma\left(\theta_{p}\right) \leq 5$, whereas subsets with 14 and more elements all have $\gamma\left(\theta_{p}\right)>5$. Therefore, we consider a maximum of 13 parameters as potentially identifiable from the available data. The subset containing the largest number of parameters with the smallest collinearity index $\gamma\left(\theta_{p}\right) \leq 5$ is selected as the best identifiable; and has collinearity index $\gamma\left(\theta_{p}\right)=4.27$.

Experience reported in the literature suggest that parameter $b_{\mathrm{PAO}}$ is difficult to estimate, even though the model is sensitive to this parameter (Brun et al. 2002): typically, an unrealistic value of $b_{\mathrm{PAO}}$ is found in the parameter estimation. We thus choose to take $b_{\mathrm{PAO}}$ out of the set of parameters to estimate. With this simplification, $\gamma\left(\theta_{p}\right)$ drops to 4.20 . The chosen final subset of 12 parameters consists of 2 parameters related to hydrolysis of particulate substrate, 3 parameters related to heterotrophic organisms, and 7 related to phosphorusaccumulating organisms; these parameters are indicated with an asterisk in Table 4.

\subsection{Parameter estimation}

After finding an identifiable parameter subset based on knowledge of $y_{t}^{m}\left(\theta^{0} \mid \mathcal{K}\right)$, we are ready to estimate the parameters. To do so, we need responses $y_{t}, t \in\{1, \ldots, T\}$ from the plant. As an initial study before doing parameter estimation based on real data from the Gävle plant, we choose to check whether suitable parameter estimates can be obtained based on responses from the simulation model. Thus, the nominal parameters in the ASM2d plant are chosen as the "correct" parameters $\theta^{*}$, and then some outputs $y_{t}(\mathcal{K})=y_{t}^{m}\left(\theta^{*} \mid \mathcal{K}\right)$ are computed. The initial parameter guess $\theta^{0}$ was chosen as a $10 \%$ increase of $\theta^{*}$. Some responses $y_{t}(\mathcal{K})$ are displayed in Figure 2 with a sludge age of $6 \mathrm{~d}$. Clearly, the outputs indicate a relatively stiff system (compare e.g. the slow variation of $S_{\mathrm{NH}_{4}}$ to the rapid variation of $S_{\mathrm{PO}_{4}}$ ), with relatively little excitation. Hence, difficulties may be expected in finding all parameters. Also, as we have seen, most of the identifiable parameters are related to the phosphorous removal, so we would expect that states related to phosphorous can be fitted better to the data.

In order to find parameter estimates, a weighted least squares criterion is applied

$$
\hat{\theta}=\arg \min _{\theta} J=\arg \min _{\theta} e^{T} W e
$$

where $\hat{\theta}$ contains the optimal parameter values of the parameters marked with an asterisk in Table 4 and the other parameters are chosen as $\theta^{0}, W$ is block diagonal with $W_{t}$ in the diagonal blocks,

$$
W_{t}=\operatorname{diag}\left(1 /\left(y_{1}^{\circ}\right)^{2}, \ldots, 1 /\left(y_{n_{y}}^{\circ}\right)^{2}\right),
$$

and $e$ is the observation error $y-y^{m}$ (Dueñas Díez et al. 2006).

To compute the parameter estimates, the nonlinear least squares algorithm 1sqnonlin of the Optimization Toolbox in Matlab is used. Table 5 shows the initial parameter values $\theta^{0}$, the "correct" values $\theta^{*}$, and the estimated values $\hat{\theta}$ obtained after minimizing $J$. 
Table 5. Comparison of initial and estimated parameter values.

\begin{tabular}{|c|c|c|c|c|c|}
\hline Symbol & Description & $\theta^{0}$ & $\hat{\theta}$ & $\theta^{*}$ & Unit \\
\hline$\mu_{\mathrm{PAO}}$ & Maximum growth rate of $\mathrm{PAO}$ & 0.0322 & 0.03156 & 0.0293 & $\mathrm{~h}^{-1}$ \\
\hline$q_{\mathrm{PP}}$ & Rate constant for storage of $X_{\mathrm{PP}}$ & 0.0385 & 0.03876 & 0.0350 & $\mathrm{~g} X_{P P} \mathrm{~g}^{-1} X_{P A O} \mathrm{~h}^{-1}$ \\
\hline$K_{\mathrm{X}}$ & $\begin{array}{l}\text { Saturation coefficient for particulate } \\
\text { COD }\end{array}$ & 0.1100 & 0.11537 & 0.1000 & 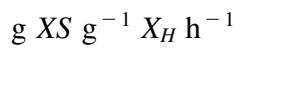 \\
\hline$\mu_{\mathrm{H}}$ & Maximum growth rate on substrate & 0.1513 & 0.16832 & 0.1375 & $\mathrm{~g} X_{S} \mathrm{~g}^{-1} X_{H} \mathrm{~h}^{-1}$ \\
\hline$q_{\mathrm{fe}}$ & Maximum rate for fermentation & 0.0756 & 0.03970 & 0.0688 & $\mathrm{~g} S_{F} \mathrm{~g}^{-1} X_{H} \mathrm{~h}^{-1}$ \\
\hline$K_{\mathrm{A}, \mathrm{PAO}}$ & Saturation coefficient for acetate, $S_{\mathrm{A}}$ & 4.4000 & 4.42080 & 4.0000 & $\mathrm{~g} \mathrm{COD} \mathrm{m}^{-3}$ \\
\hline$q_{\mathrm{PHA}}$ & $\begin{array}{l}\text { Rate constant for storage of } X_{\mathrm{PHA}} \\
\quad \text { (base } X_{\mathrm{PP}} \text { ) }\end{array}$ & 0.0963 & 0.10698 & 0.0875 & $\mathrm{~g} X_{P H A} \mathrm{~g}^{-1} X_{P A O} \mathrm{~h}^{-1}$ \\
\hline$K_{\mathrm{PHA}}$ & Saturation coefficient for PHA & 0.0110 & 0.01125 & 0.0100 & $\mathrm{~g} X_{P H A} \mathrm{~g}^{-1} X_{P A O}$ \\
\hline$b_{\mathrm{HET}}$ & Rate for Lysis of $X_{\mathrm{H}}$ & 0.0101 & 0.01049 & 0.0092 & $\mathrm{~h}^{-1}$ \\
\hline$\eta_{\mathrm{fe}}$ & Anoxic hydrolysis reduction factor & 0.4400 & 0.45286 & 0.4000 & - \\
\hline$K_{\mathrm{PS}, \mathrm{PAO}}$ & $\begin{array}{l}\text { Saturation coefficient for phosphorus } \\
\text { in storage of PP }\end{array}$ & 0.2200 & 0.21772 & 0.2000 & $\mathrm{~g} \mathrm{P} \mathrm{m}^{-3}$ \\
\hline$b_{\mathrm{PP}}$ & Rate for Lysis of $X_{\mathrm{PP}}$ & 0.0050 & 0.00513 & 0.0046 & $\mathrm{~h}^{-1}$ \\
\hline
\end{tabular}

Note that for several parameters, $\left|\theta_{j}^{*}-\theta_{j}^{0}\right|<\left|\theta_{j}^{*}-\hat{\theta}_{j}\right|$. The reason for this is that we are estimating only a subset of the parameters.

\subsection{Model validation}

Validation is the comparison of model output $y_{t}^{m}\left(\hat{\theta} \mid \mathcal{K}^{\prime}\right)$ with the real output $y_{t}\left(\mathcal{K}^{\prime}\right)$ based on validation data $\mathcal{K}^{\prime}$, while parameter estimate $\hat{\theta}$ is based on training data $\mathcal{K}$. The main reason why the validation data $\mathcal{K}^{\prime}$ should differ from the training data $\mathcal{K}$ is to avoid fitting the model to noise. In this paper, we constructed the training data from an assumed perfect model, $y_{t}=y_{t}^{m}\left(\theta^{*} \mid \mathcal{K}\right)$, and we choose to use the training data for validation, too. The evolution of some key outputs are displayed in Figure 3: the "real" output $y_{t}=y_{t}^{m}\left(\theta^{*}\right)$ from the biological reactor, and the prediction outputs $\hat{y}_{t}=y_{t}^{m}(\hat{\theta})$. For comparison, we also include the model outputs $y_{t}^{m}\left(\theta^{0}\right)$, which will indicate how much the model has been improved through parameter estimation.

The concentration of $\mathrm{S}_{\mathrm{PO}_{4}}$ after parameter estimation (dashed red curve in Figure 3) is quite close to the "real" process output (black, solid line). As argued previously, the model simulates bio-P operation, which can be observed from the time evolution of $S_{\mathrm{PO}_{4}}$. We also argued that due to the bio-P operation, most of the identifiable parameters are related to phosphorous removal. Furthermore, more weight has been put on phosphorous removal outputs because of the governmental restrictions on total phosphorous in the effluent. Thus, the model has mainly been adjusted to give good prediction of those measurements which are related to phosphorous.

The prediction capabilities of other outputs (e.g. $S_{\mathrm{NH}_{4}}$ and $S_{\mathrm{NO}_{3}}$ ) are poorer. As argued, the lack of autotrophic biomass $\left(X_{\mathrm{AUT}}\right)$ in the process leads to poor nitrification. Consequently, parameters related to the autotrophic (nitrifying) organism group are poorly identifiable. However, since there are no government restrictions on the nitrogen outputs, the poor prediction capability of nitrogen outputs is acceptable. 

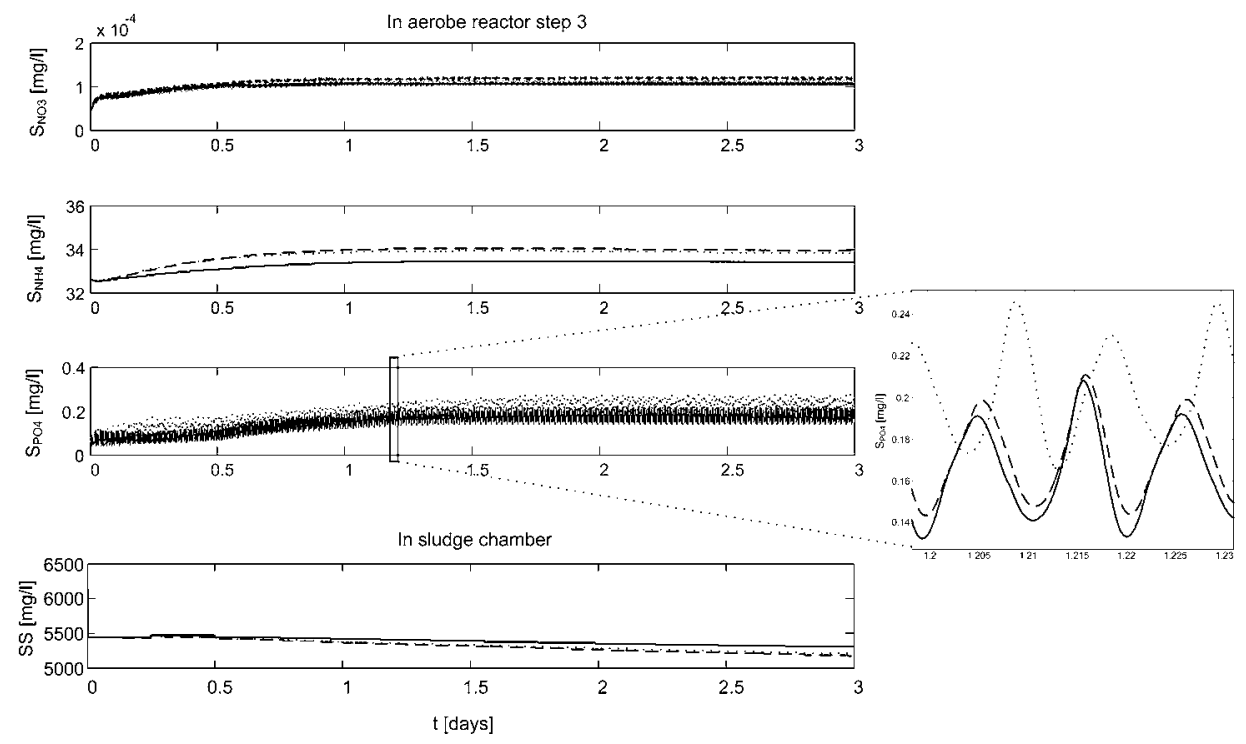

Figure 3. Comparison of model outputs: $y_{t}=y_{t}^{m}\left(\theta^{*}\right)$ (solid, black), $\hat{y}_{t}=y_{t}^{m}(\hat{\theta})$ (dashed, red), and $y_{t}^{m}\left(\theta^{0}\right)$ (dotted, blue).

\section{Conclusions}

In this paper, a method for assessing parameter identifiability of large scale models based on experimental data is discussed. The method is applied to a model of a biological wastewater treatment plant. First, a general description of wastewater treatment is given, with an introduction to the ASM2d dynamic model. The description is unique in the sense that it does not require much background in biological waste water modeling, apart from a background in basic process modeling. Next, an overview is given of a method for analyzing experimental identifiability which has been proposed in the literature. Then, an overview of the Duvbacken wastewater treatment plant in Gävle, Sweden, is given. Finally, the identifiability analysis theory is applied to a model of the Duvbacken plant. It should be emphasized that because real experimental data were unavailable, the study has been based on simulated data assuming a perfect model. However, the procedure can be used without modification with real data.

In the analysis, a model with 45 parameters has been studied. From the experimental identifiability analysis, 12 of the parameters were found to be identifiable. Some details in the analysis are given. Then these 12 parameters are used in a parameter estimation study, and improved parameters are found using a Matlab routine for nonlinear least squares minimization. After finding the parameters, the model is validated against the original experimental data. The model fit is not perfect. However, the results are logical from an understanding of the process operation: the model fit is quite good for those outputs that matter in the operation of the plant, and less good in outputs which are less important to the plant operation.

Some future work may include:

- The current model implementation in Matlab is relatively rigid with respect to possible input signals, and a rewriting of the model in a more flexible modeling language such as Modelica is planned. 
- Verifying the model more thoroughly for other operating scenarios.

- Fitting the model to experimental data for larger initial errors in parameters, and comparing different optimization algorithms, as well as multiple shooting vs. single shooting (used here).

- Fitting the model to noisy data/real experimental data.

- Including a statistical analysis of the parameters and prediction capabilities of the model.

\section{References}

Bailey, J. E. \& Ollis, D. F. (1986). Biochemical Engineering Fundamentals, second edn, McGraw-Hill, New York.

BARD, Y. (1974). Nonlinear Parameter Estimation, Academic Press, New York.

BeCK, J. V. \& ARNOLD, K. J. (1977). Parameter Estimation in Engineering and Science, Wiley, New York.

Belsley, D. A. (1991). Conditioning Diagnostics. Collinearity and Weak Data in Regression, John Wiley \& Sons, New York.

Brun, R., Kühni, M., Siegrist, H., Gujer, W. \& Reichert, P. (2002). 'Practical identifiability of ASM2d parameters-systematic selection and tuning of parameter subsets', Water Research 36(16), 4113-4127.

Brun, R., ReICHERT, P. \& KüNSCH, H. R. (2001). 'Practical identifiability analysis of large environmental simulation models', Water Resour. Res. 37(4), 1015-1030.

DueÑas Díez, M., FJeld, M., Andersen, E. \& Lie, B. (2006). 'Validation of a compartmental population balance model of an industrial leaching process: The Silgrain(R) process', Chem. Engng. Sci. 61, 229-245.

Henze, M., Gujer, W., Mino, T., Matsuo, T., Wentzel, M. C., Marais, G. V. R. \& van LoosDRECHT, M. C. M. (1999). 'Activated sludge model no. 2d, ASM2D', Wat. Sci. Tech. 39(1), $165-182$.

Henze, M., GuJer, W., van Loosdrecht, M. C. M. \& Mino, T. (2000). Activated Sludge Models ASM1, ASM2, ASM2d and ASM3., Vol. 9 of Scientific and Technical Report, IWA Publishing.

Henze, M., Harremoës, P., la Cour Jansen, J. \& Arvin, E. (1996). Wastewater Treatment. Biological and Chemical Processes, second edn, Springer, Berlin.

Holmberg, A. (1982). 'On the parameter identifiability of microbial growth models incorporating michaelesmenten type nonlinearities', Math. Biosci. 62, 23-43.

JEPPSON, U. (1993). On the verifiability of the activated sludge system dynamics, Tekn. lic. dissertation, Lund Institute of Technology.

LuUnG, L. (1999). System Identification. Theory for the User, second edn, Prentice Hall, Upper Saddle River, New Jersey.

LJUNG, L. \& GlaD, S. T. (1994). 'On global identifiability for arbitrary model parameterizations', Automatica 30(2), 265-276.

Metcalf \& EdDY, I. (2003). Wastewater Engineering. Treatment and Reuse, fourth edn, McGraw Hill, New York.

Olsson, G. \& Newell, B. (1999). Wastewater Treatment Systems. Modelling, Diagnosis and Control, IWA Publishing, London.

Pohjanpalo, H. (1978). 'System Identifiability Based on the Power Series Expansion of the Solution', Mathematical Biosciences 41, 21-33.

Rawlings, J. B. \& Ekerdt, J. G. (2002). Chemical Reactor Analysis and Design Fundamentals, Nob Hill Publishing, Madison, Wisconsin.

Smith, W. R. \& Missen, R. W. (1991). Chemical Reaction Equilibrium Analysis: Theory and Algorithms, Krieger Publishing Company, Malabar, Florida.

Söderström, T. \& Stoica, P. (1989). System Identification, Prentice Hall International.

Stewart, G. (1987), 'Collinearity and least squares regression', Statistical Science 2(1), 68-84.

Walter, E. \& Pronzato, L. (1997). Identification of Parametric Models from Experimental Data, Springer. 